Research Article

\title{
Theoretical Study of the Effect of Instrument Parameters on the Flow Field of Air-Flow Impacting Based Mechanochemical Synthesis
}

\author{
Yang Tao $(\mathbb{D}$, Jun Lin, Zhao Zhang, Qiuting Guo, Jin Zuo, and Bo Lu \\ High Speed Aerodynamics Institute, China Aerodynamic Research and Development Center, Mianyang 621000, China \\ Correspondence should be addressed to Yang Tao; 50323222@qq.com
}

Received 3 January 2018; Revised 20 March 2018; Accepted 25 March 2018; Published 24 May 2018

Academic Editor: Jose C. Merchuk

Copyright (c) 2018 Yang Tao et al. This is an open access article distributed under the Creative Commons Attribution License, which permits unrestricted use, distribution, and reproduction in any medium, provided the original work is properly cited.

\begin{abstract}
The air-flow impacting based mechanochemical synthesis is an alternative strategy to traditional mechanochemical preparations, which has many advantages in terms of reaction temperature, preparation speed, and cleanness. Herein, we theoretically study the effect of instrument parameters, including the axial position of physical target, the diameter difference between nozzle throat and suction pipe, and divergence angles of uniform speed region, on the flow field of the air-flow impacting based mechanochemical synthesis. The optimized parameters have been obtained. Under the optimal conditions, a stable and high-speed air flow is obtained, in which the speed can achieve a Mach number of approximately 2.6. The high-speed air flow is able to easily carry the reacting substances to arrive at the physical target, triggering a chemical reaction. These findings undoubtedly provide a key guideline for further development and application of the air-flow impacting based mechanochemical synthesis.
\end{abstract}

\section{Introduction}

The mechanochemical synthesis has received more and more attention due to its unique advantages such as simpleness, economy, and environmental friendliness [1]. It can facilely proceed in the solid state without the use of solvents. A variety of substances spanning from organic compounds to metal-organic frameworks to nanomaterials have been successfully prepared using the mechanochemical synthesis techniques [1-7]. Traditionally, the mechanochemical synthesis is carried out by hand grinding, ball milling, and twin screw extrusion [8-14]. However, these methods suffer from many drawbacks. For instance, these methods will produce a high temperature during the procedures, which are not suitable to prepare thermally sensitive compounds. Additionally, the preparation speed is limited at $\mathrm{kg} \cdot \mathrm{h}^{-1}$, making them insufficient to realize the large-scale synthesis.

In order to overcome these shortcomings, we recently developed a novel air-flow impacting based mechanochemical synthesis [15]. In this strategy, the air flow can be accelerated to a maximum velocity going up to $300-600 \mathrm{~m} / \mathrm{s}$ at room temperature (approximately $25^{\circ} \mathrm{C}$ ). When the reacting substances are carried by the air flow with a supersonic speed, they will tempestuously collide with each other or a physical target, accompanying that the mechanical energy effectively initiates the chemical reaction. The gaseous medium is employed instead of physical ball, pestle, and screw, resulting in a more clean and renewable synthesis. More importantly, it has been demonstrated that the air-flow impacting based mechanochemical synthesis is able to achieve large-scale and fast preparation at $\mathrm{kg} \cdot \mathrm{min}^{-1}$ rates, which is at least sixty times faster than the traditional mechanochemical synthesis methods. Obviously, the performance of air-flow impacting based mechanochemical synthesis is highly dependent on the air-flow field in the instrument. To better understand the preparation process, it is highly desirable to study the air-flow field in the instrument.

Here, we theoretically investigate the effect of aerodynamic design parameters of the instrument on the flow field of the air-flow impacting based mechanochemical synthesis. Several instrument parameters, including the physical target, the diameter difference between nozzle throat inwall and 
suction pipe of solid reaction particles, and divergence angles of acceleration pipe, have been investigated. Based on the analysis results, we obtain the optimized aerodynamic design parameters. This work provides a guideline for the air-flow impacting based mechanochemical synthesis.

\section{Methods}

2.1. Design of De Laval Nozzle. De Laval nozzle is employed to produce a high-speed air flow with a supersonic speed. Isentropic flow was assumed in the annular nozzle. The produced Mach number $\left(M_{\mathrm{a}}\right)$ is depended on the area ratio of exit surface and throat $A_{\mathrm{ex}} / A_{\mathrm{thr}}$, according to the following equation $[16,17]$ :

$$
\frac{A_{\mathrm{ex}}}{A_{\mathrm{thr}}}=\frac{1}{M_{\mathrm{a}}}\left(\frac{2}{\gamma+1}\left(1+\frac{\gamma-1}{2} M_{\mathrm{a}}^{2}\right)\right)^{(\gamma+1) /(2(\gamma-1))},
$$

where $\gamma$ is the ratio of specific heats, which is taken to be constant of 1.4 for the diatomic gas molecules, $A_{\mathrm{ex}}$ is the area of the exhaust, and $A_{\mathrm{thr}}$ is the area of the throat.

The pressure ratio of the total pressure to the static pressure at the intake side and exhaust side must maintain a high enough value, and the critical pressure ratio can be detected by the following relationships [18-20]:

$$
\frac{p_{0}}{p_{\mathrm{ex}}}=\left(\frac{T_{0}}{T_{\mathrm{ex}}}\right)^{\gamma /(\gamma-1)}=\left(1+\frac{\gamma-1}{2} M_{\mathrm{a}}^{2}\right)^{\gamma /(\gamma-1)},
$$

where $p_{0}$ and $T_{0}$ are the total pressure and total temperature at intake side and $p_{\mathrm{ex}}$ and $T_{\mathrm{ex}}$ are the static pressure and static temperature at the exhaust side of the nozzle.

Also, the various parts of the De Laval nozzle, including converge section, diverge section, and uniform speed section, are calculated to obtain the physical appearance such as size, length, and angle through a series of equations as shown in the supporting information (available here). To ensure that the theoretical Mach number can reach 3, the physical sizes of the De Laval nozzle are as follows: converge section (length: $40 \mathrm{~mm}$, diameter: $9.26 \mathrm{~mm}$ ), throat diameter $(3 \mathrm{~mm})$, and diverge section (length: $24.69 \mathrm{~mm}$, diameter: $6.17 \mathrm{~mm})$.

2.2. Numerical Simulation. We employ the commercial CFD code Fluent 6.3 for the numerical simulation based on the above-mentioned De Laval nozzle and variable instrumental parameters as mentioned in our manuscript. The computational analysis uses the Navier-Stokes equations, which can be written in a conservational form as follows [21]:

$$
\begin{aligned}
\frac{\partial \rho}{\partial t}+\frac{\partial}{\partial x_{j}}\left(\rho u_{j}\right) & =0 \\
\frac{\partial\left(\rho u_{i}\right)}{\partial t}+\frac{\partial}{\partial x_{j}}\left(\rho u_{i} u_{j}+\delta_{i j} p\right) & =\frac{\partial \tau_{i j}}{\partial x_{j}} \\
\frac{\partial(\rho E)}{\partial t}+\frac{\partial}{\partial x_{j}}\left(\rho H u_{j}\right) & =\frac{\partial}{\partial x_{j}}\left(\tau_{i j} u_{i}+\lambda \frac{\partial T}{\partial x_{j}}\right),
\end{aligned}
$$

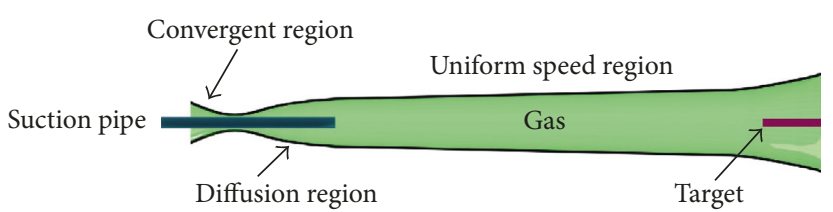

FIGURE 1: Schematic representation of the air-flow impacting based mechanochemical synthesis instrument.

where $\rho, p$, and $T$ denote flow density, pressure, and temperature, respectively; $u_{j}$ is the velocity component along the Cartesian coordinate $x_{j} ; E$ and $H$ are the total energy and the total enthalpy per unit volume, respectively; $\tau_{i j}$ is the stress tensor; $\lambda$ is the heat transfer coefficient; and $\delta_{i j}$ is the Kronecker delta. The equation of state of ideal gas is introduced to close the system.

The RANS equations with the $k-\omega$ SST turbulence model are used for the computational analysis. The SST turbulence model is employed for high-accuracy boundary-layer simulation. It is a hybrid method that couples the standard $k-\varepsilon$ and $k$ - $\omega$ models in an efficient manner, with the $k$ - $\omega$ model used in the near-wall region and the standard $k-\varepsilon$ model in the far-field region, blending them together at the interface between the regions. It includes the modeling of transport of shear stress via a modified definition of the turbulent viscosity.

The pressure inlet boundary conditions were used at the intakes of the nozzle and suction pipe. The pressure outlet boundary condition was applied at the exit. The no-slipped wall was used for all of the solid walls. Moreover, all walls were assumed as ideal rigid solid surfaces without deformation during the collision. The overall analysis of the flow field of air-flow impacting based mechanochemical synthesis was carried out on ANSYS 16.0.

\section{Results and Discussion}

3.1. The Structure of the Air-Flow Impacting Instrument. As shown in Figure 1, the air-flow impacting instrument is consisting of suction pipe, convergent region, diffusion region, uniform speed region, and a physical target. When compressed air is injected into this instrument, the air is accelerated to a subsonic speed in the convergent region. The speed of the air is further increased to the supersonic speed in the diffusion region. The high-speed air can carry the reacting substances to collide with a physical target, triggering a chemical reaction. The speed of the air is a key factor for the chemical preparation, which is mainly dependent on the diameter difference between nozzle throat and suction pipe, the position of the physical target, and the divergence angles.

\subsection{Optimization of Aerodynamic Design Parameters for the} Flow Field. To describe the speed of the air flow, the Mach number is introduced, which is expressed as $M_{\mathrm{a}}=u / c$, where $u$ is the local flow velocity with respect to the boundaries and $c$ represents the speed of sound in the medium. It can be expressed as $c=\sqrt{\gamma R T}$, where $\gamma$ is the adiabatic exponent of 

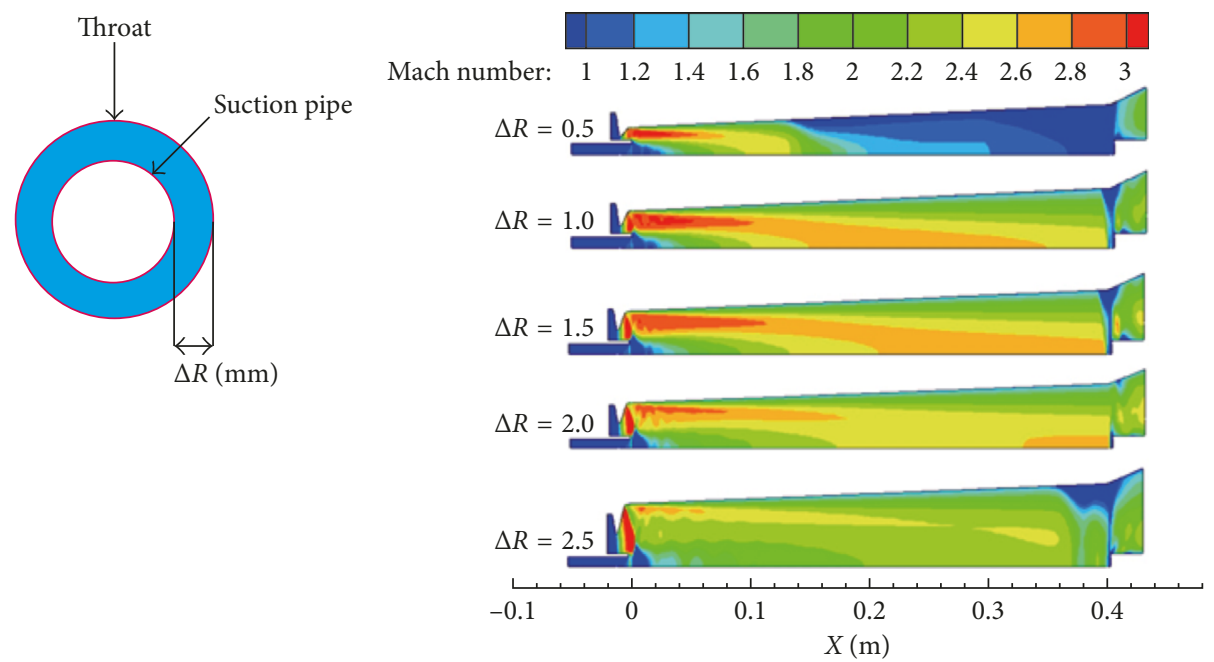

(a)

(b)

FIGURE 2: (a) The front view of the throat and suction pipe. (b) Effect of the diameter difference between nozzle throat and suction pipe ( $\Delta R$ ) on the flow field of the air flow.
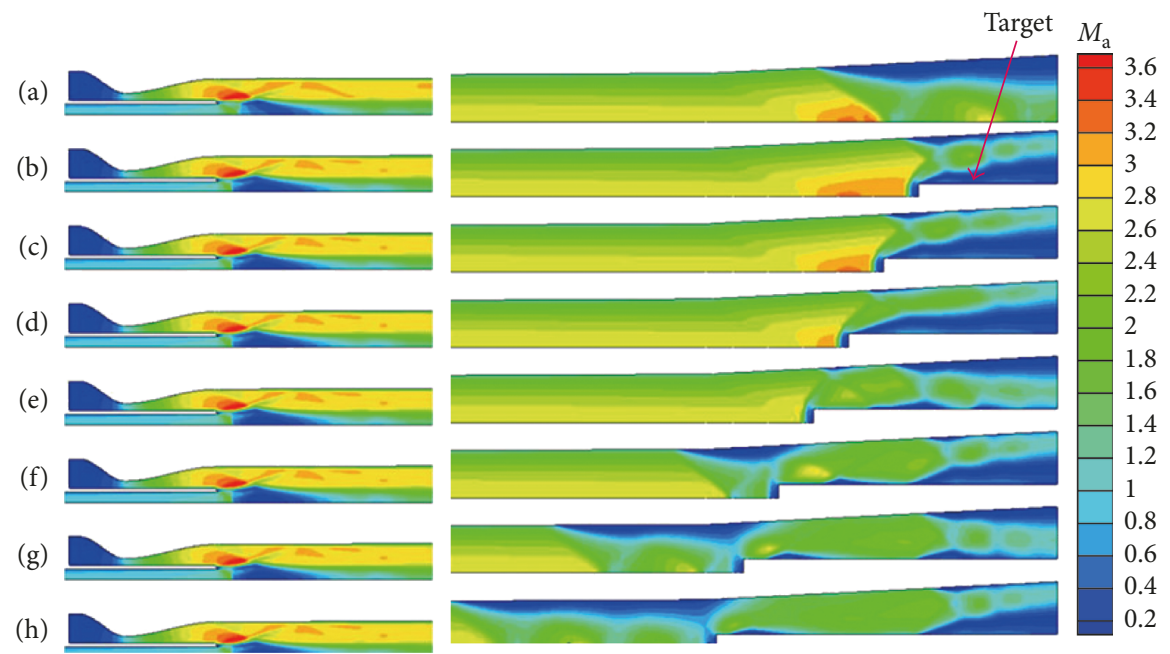

FIGURE 3: Cross-sectional flow field of air flow in the absence (a) and presence of a physical target placed under different positions: (b) 410, (c) 405, (d) 400, (e) 395, (f) 390, (g) 385, and (h) $381 \mathrm{~mm}$.

air, $R$ is the gas constant, and $T$ is the thermodynamic temperature [22]. We can solve the Navier-Stokes equations (5) to obtain the $T$ at different positions, which are further employed for calculating $c$. The effect of the diameter difference between nozzle throat and suction pipe $(\Delta R)$ on the flow field is firstly investigated. As shown in Figure 2, when the value of the $\Delta R$ is lower than $0.5 \mathrm{~mm}$, the speed of the compressed air quickly decreases to a sonic speed after spraying. Additionally, if the $\Delta R$ is above $2.5 \mathrm{~mm}$, the air flow is apt to form a curved shock wave, which is unstable (Figure 2(b)). Therefore, in order to obtain a stable high-speed air flow, the value of the $\Delta R$ should be kept in the range of $1.0-2.0 \mathrm{~mm}$.

Apart from the diameter difference between throat inwall and suction pipe $\Delta R$, the axial position of the physical target apart from the spraying outlet is another important factor. As displayed in Figure 3(a), it cannot obtain a stable flow field in the absence of the physical target, and the speed of the air flow is relatively low. As the physical target is placed in the instrument, a stable and high-speed air flow can be obtained as shown in Figures 3(b)-3(e). Nevertheless, the physical target is also able to disturb the flow field of the air flow if it is too near the spraying outlet (Figures 3(f)-3(h)). Simultaneously, under this condition, it can result in local congestion, leading to moving the curved shock wave forward. While the distance between the physical target and the spraying outlet is in the range from 395 to $410 \mathrm{~mm}$, a stable and high-speed air flow can be obtained, and the average of $M_{\mathrm{a}}$ is approximately 2.6. Therefore, the optimized axial distance between the physical target and the spraying outlet is ranging from 395 to $410 \mathrm{~mm}$. 


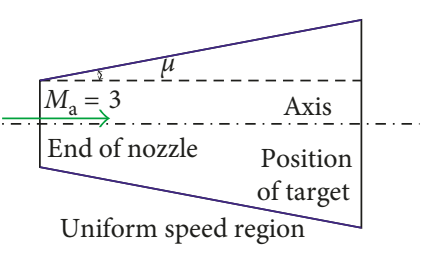

(a)

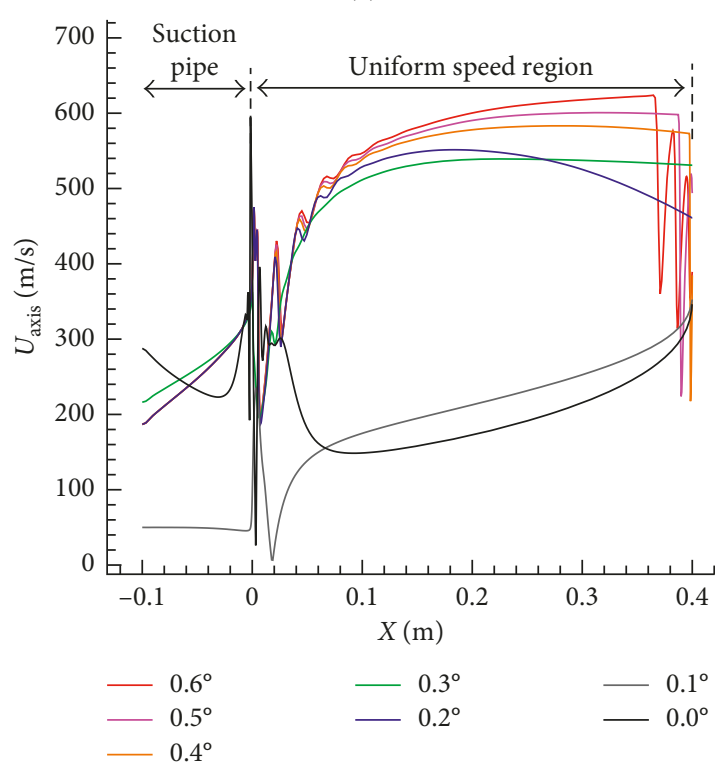

(b)

Figure 4: (a) The scheme for the divergence angle $(\mu)$. (b) The relationship between the axis speed $\left(U_{\text {axis }}\right)$ and the divergence angle from $0^{\circ}$ to $0.6^{\circ}$ in the uniform speed region.

In order to avoid the appearance of strong shock wave and keep a high air flow, the divergence angle is introduced in the uniform speed region as shown in Figure 4(a). The effect of the divergence angle on the axial speed $\left(U_{\text {axis }}\right)$ is examined at a $M_{\mathrm{a}}$ of 3 . The high $M_{\mathrm{a}}$ can promote the collisional processes, which effectively initiates the chemical reaction. In the present stage, it is very difficult to achieve the equipment with the $M_{\mathrm{a}}$ number of more than 3 because of the limitations in terms of materials, carrier gas, and pressure. It can be seen that the absence of the divergence angle is able to result in gas congestion and a fast decrease of the $U_{\text {axis }}$, which decreases to the subsonic speed. This result confirms that the introduction of the divergence angle is quite necessary. In addition, with the increasing the divergence angle from $0.1^{\circ}$ to $0.3^{\circ}$, the value of the $U_{\text {axis }}$ gradually increases and reaches a stable value at $0.3^{\circ}$. However, when the divergence angle is larger than $0.3^{\circ}$, the unstable shock wave appears in the end of the uniform speed region. Accordingly, the optimized divergence angle is $0.3^{\circ}$ to obtain a stable and high-speed air flow.

3.3. Distribution of Air-Flow Field. Under the optimal aerodynamic design parameters, we further investigate the distribution of air-flow field in the air-flow impacting based mechanochemical synthesis instrument. As indicated in

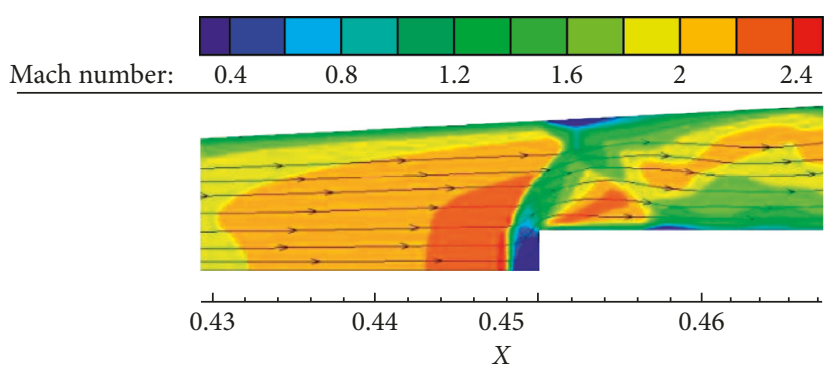

(a)

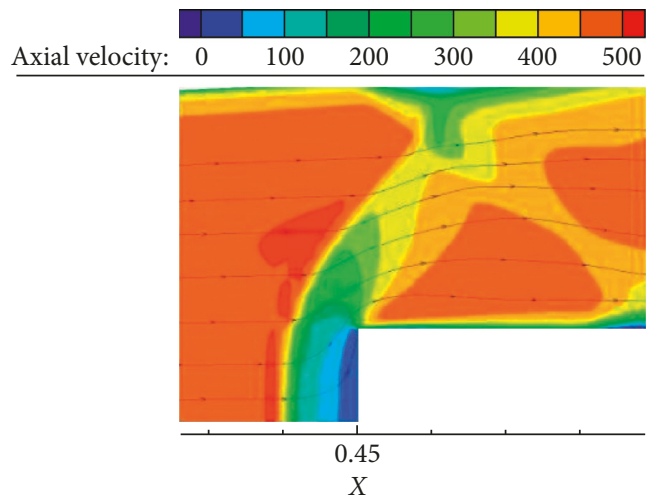

(b)

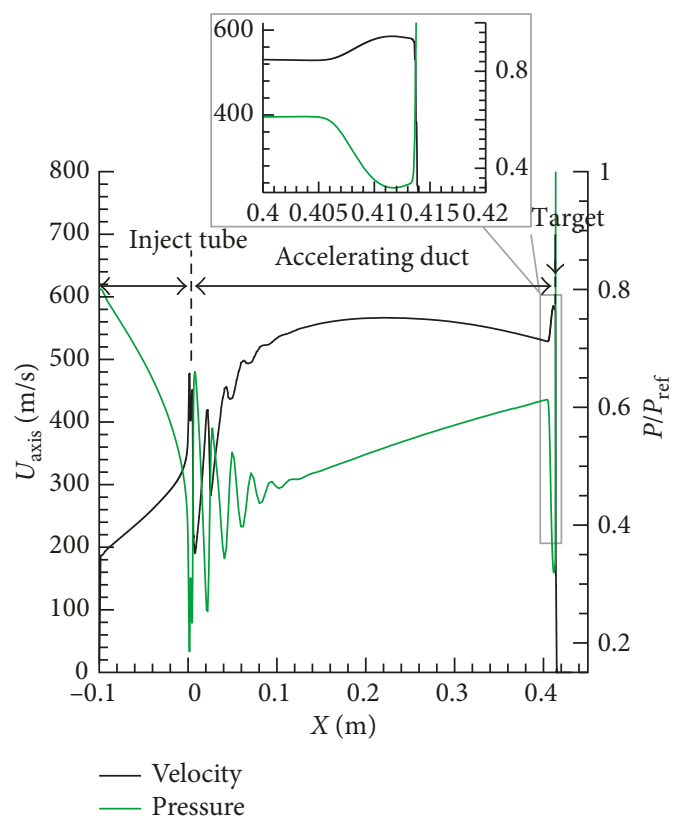

(c)

Figure 5: $(a, b)$ The distribution of air-flow field at the diffusion region and the physical target. (c) The velocity and pressure versus the position at the axis line, where $P$ and $P_{\text {ref }}$ represent the local static pressures and reference pressure with mean value of $1 \mathrm{~atm}$.

Figure 5, the high pressure gas is accelerated in the convergent region, and the gas achieves a relatively high speed. Besides, an extremely relative static pressure $\left(P / P_{\text {ref }}=0.1\right.$, where $P$ and $P_{\text {ref }}$ are the local static pressures and reference pressure with mean value of $1 \mathrm{~atm}$, resp.) is obtained in the exit of the suction pipe. The great pressure difference is in 
favor of the injection of various reaction substances into the reaction instrument. Furthermore, the low-speed air flow from the suction pipe is carried by the air flow with a high speed in the diffusion region, which crosses over the uniform speed region. Eventually, a high-speed air flow and strong bow shock wave are formed near the physical target. The maximum speed of the air flow is about $550 \mathrm{~m} / \mathrm{s}$ with a local $M_{\mathrm{a}}$ of 2.6, which is capable of supplying mechanochemical energy for powerful effective chemical preparation.

\section{Conclusions}

In summary, we investigate the effect of the instrument parameters, such as the physical target, the diameter difference between throat inwall and suction pipe, and the divergence angles, on the flow field of air-flow impacting based mechanochemical synthesis. The results illustrate that these parameters strongly affect the speed of air flow. The average $M_{\mathrm{a}}$ of the air flow can reach 2.6. Additionally, the air flow with a high speed forms a stable flow field, which can carry the reaction substances to collide with the physical target, inducing a chemical reaction. These findings may serve as a guide to the air-flow impacting based mechanochemical synthesis and further broaden the application field of the mechanochemical synthesis.

\section{Data Availability}

The data used to support the findings of this study are available from the corresponding author upon request.

\section{Conflicts of Interest}

The authors declare that there are no conflicts of interest regarding the publication of this paper.

\section{Acknowledgments}

The support of this research by the National Natural Science Foundation of China (Grant no. 51327804) is gratefully acknowledged.

\section{Supplementary Materials}

Figure S1: the structure of De Laval nozzle. Figures S2 and S3: the corresponding theoretical calculations. (Supplementary Materials)

\section{References}

[1] B. P. Biswal, S. Chandra, S. Kandambeth, B. Lukose, T. Heine, and R. Banerjee, "Mechanochemical synthesis of chemically stable isoreticular covalent organic frameworks," Journal of the American Chemical Society, vol. 135, no. 14, pp. 5328-5331, 2013.

[2] S. L. James, C. J. Adams, C. Bolm et al., "Mechanochemistry: opportunities for new and cleaner synthesis," Chemical Society Reviews, vol. 41, no. 1, pp. 413-447, 2012.

[3] G. Bharath, R. Madhu, S.-M. Chen, V. Veeramani, D. Mangalaraj, and N. Ponpandian, "Solvent-free mechanochemical synthesis of graphene oxide and $\mathrm{Fe}_{3} \mathrm{O}_{4}$-reduced graphene oxide nanocomposites for sensitive detection of nitrite," Journal of Materials Chemistry A, vol. 3, no. 30, pp. 15529-15539, 2015.

[4] P. A. Julien, K. Užarević, A. D. Katsenis et al., "In situ monitoring and mechanism of the mechanochemical formation of a microporous MOF-74 framework," Journal of the American Chemical Society, vol. 138, no. 9, pp. 2929-2932, 2016.

[5] D. Prochowicz, K. Sokołowski, I. Justyniak et al., "A mechanochemical strategy for IRMOF assembly based on predesigned oxo-zinc precursors," Chemical Communications, vol. 51, no. 19, pp. 4032-4035, 2015.

[6] G.-W. Wang, "Mechanochemical organic synthesis," Chemical Society Reviews, vol. 42, no. 18, pp. 7668-7700, 2013.

[7] A. D. Katsenis, A. Puskaric, V. Strukil et al., "In situ X-ray diffraction monitoring of a mechanochemical reaction reveals a unique topology metal-organic framework," Nature Communications, vol. 6, no. 1, p. 6662, 2015.

[8] M. Y. Masoomi, A. Morsali, and P. C. Junk, "Rapid mechanochemical synthesis of two new Cd(II)-based metal-organic frameworks with high removal efficiency of Congo red," CrystEngComm, vol. 17, no. 3, pp. 686-692, 2015.

[9] I. Y. Jeon, S. Y. Bae, J. M. Seo, and J. B. Baek, "Scalable production of edge-functionalized graphene nanoplatelets via mechanochemical ball-milling," Advanced Functional Materials, vol. 25, no. 45, pp. 6961-6975, 2015.

[10] X. Ma, W. Yuan, S. E. Bell, and S. L. James, "Better understanding of mechanochemical reactions: Raman monitoring reveals surprisingly simple 'pseudo-fluid' model for a ball milling reaction," Chemical Communications, vol. 50, no. 13 , pp. 1585-1587, 2014.

[11] D. E. Crawford, L. A. Wright, S. L. James, and A. P. Abbott, "Efficient continuous synthesis of high purity deep eutectic solvents by twin screw extrusion," Chemical Communications, vol. 52, no. 22, pp. 4215-4218, 2016.

[12] D. E. Crawford, C. K. G. Miskimmin, A. B. Albadarin, G. Walker, and S. L. James, "Organic synthesis by twin screw extrusion (TSE): continuous, scalable and solvent-free," Green Chemistry, vol. 19, no. 6, pp. 1507-1518, 2017.

[13] W. Huang, Z. Xie, Y. Deng, and Y. He, " $3,3^{\prime}, 5,5^{\prime}$ tetramethylbenzidine-based quadruple-channel visual colorimetric sensor array for highly sensitive discrimination of serum antioxidants," Sensors and Actuators B: Chemical, vol. 254, pp. 1057-1060, 2018.

[14] R. Li, H. An, W. Huang, and Y. He, "Molybdenum oxide nanosheets meet ascorbic acid: tunable surface plasmon resonance and visual colorimetric detection at room temperature," Sensors and Actuators B: Chemical, vol. 259, pp. 59-63, 2018.

[15] B. Sun, Y. He, R. Peng, S. Chu, and J. Zuo, "Air-flow impacting for continuous, highly efficient, large-scale mechanochemical synthesis: a proof-of-concept study," ACS Sustainable Chemistry \& Engineering, vol. 4, no. 4, pp. 2122-2128, 2016.

[16] S. He, Y. Li, and R. Z. Wang, "Progress of mathematical modeling on ejectors," Renewable and Sustainable Energy Reviews, vol. 13, no. 8, pp. 1760-1780, 2009.

[17] N. H. Aly, A. Karameldin, and M. M. Shamloul, "Modelling and simulation of steam jet ejectors," Desalination, vol. 123, no. 1, pp. 1-8, 1999.

[18] Y. A. Çengel and M. A. Boles, Thermodynamics: An Engineering Approach, McGraw-Hill, New York, NY, USA, 8th edition, 2015, ISBN 978-0-07-339817-4.

[19] J. R. Partington, An Advanced Treatise on Physical Chemistry, Fundamental Principles. The Properties of Gases, Vol. 1, Longmans, Green \& Co., London, UK, 1949. 
[20] J. Fan, J. Eves, H. M. Thompson et al., "Computational fluid dynamic analysis and design optimization of jet pumps," Computers \& Fluids, vol. 46, no. 1, pp. 212-217, 2011.

[21] F. R. Menter, "Two-equation eddy-viscosity turbulence models for engineering applications," AIAA Journal, vol. 32, no. 8, pp. 1598-1605, 1994.

[22] V. L. Streeter and E. B. Wylie, Fluid Mechanics, McGraw-Hill Higher Education, New York, NY, USA, 9th edition, 1988. 


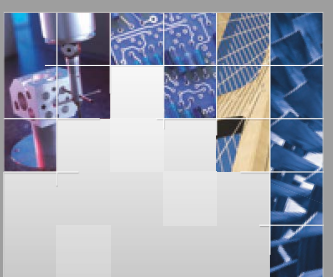

\section{Enfincering}
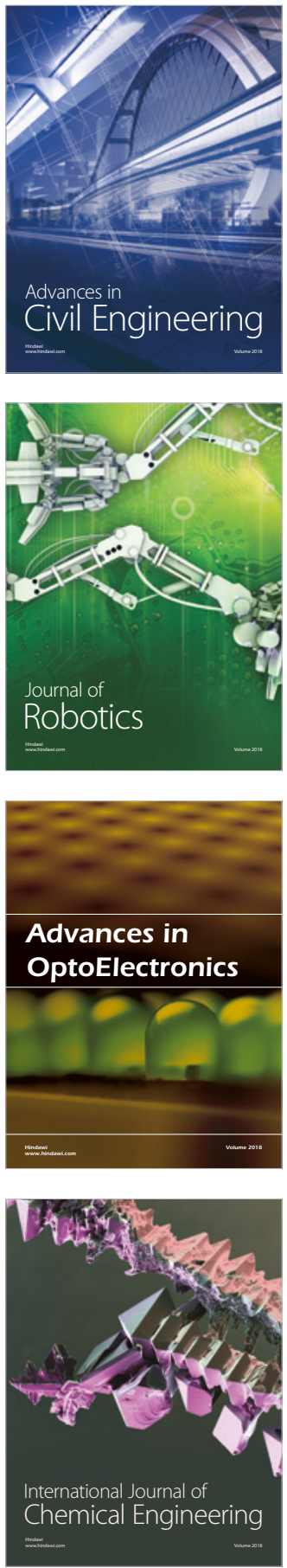

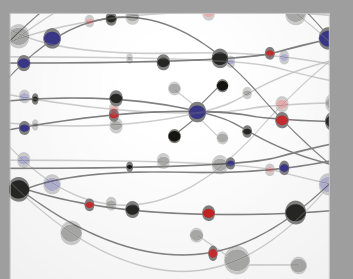

\section{Rotating \\ Machinery}

The Scientific World Journal

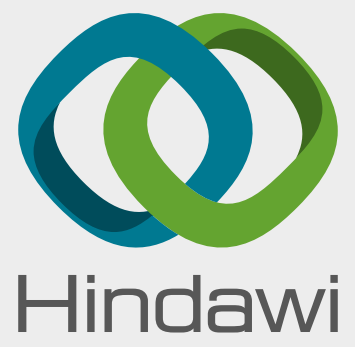

Submit your manuscripts at

www.hindawi.com
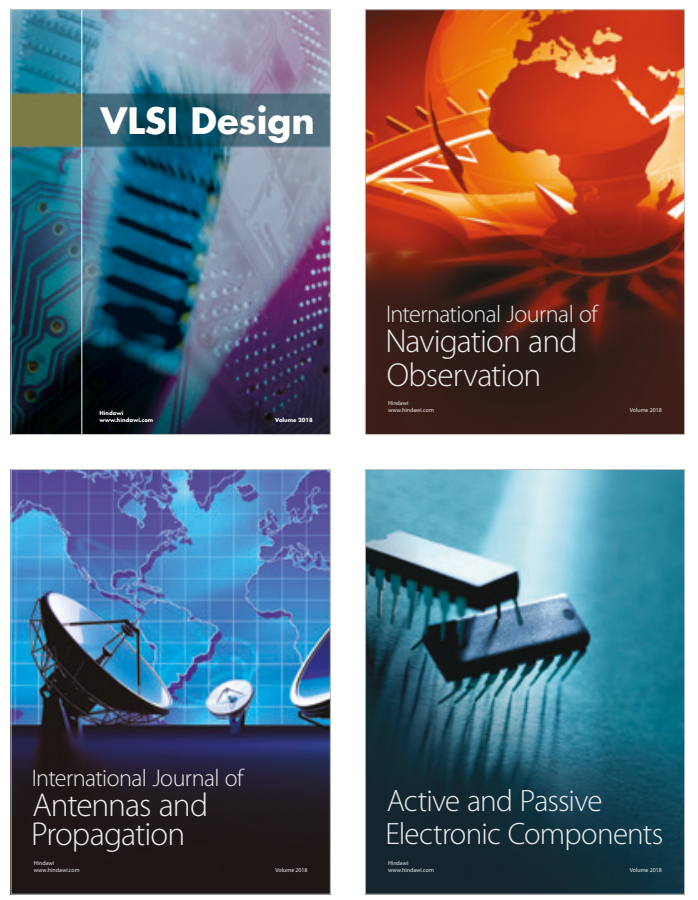
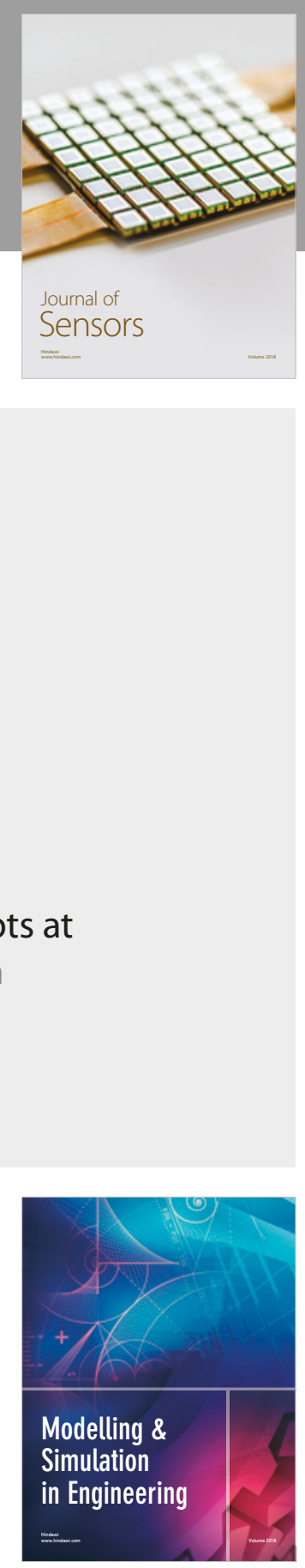

\section{Advances \\ Multimedia}
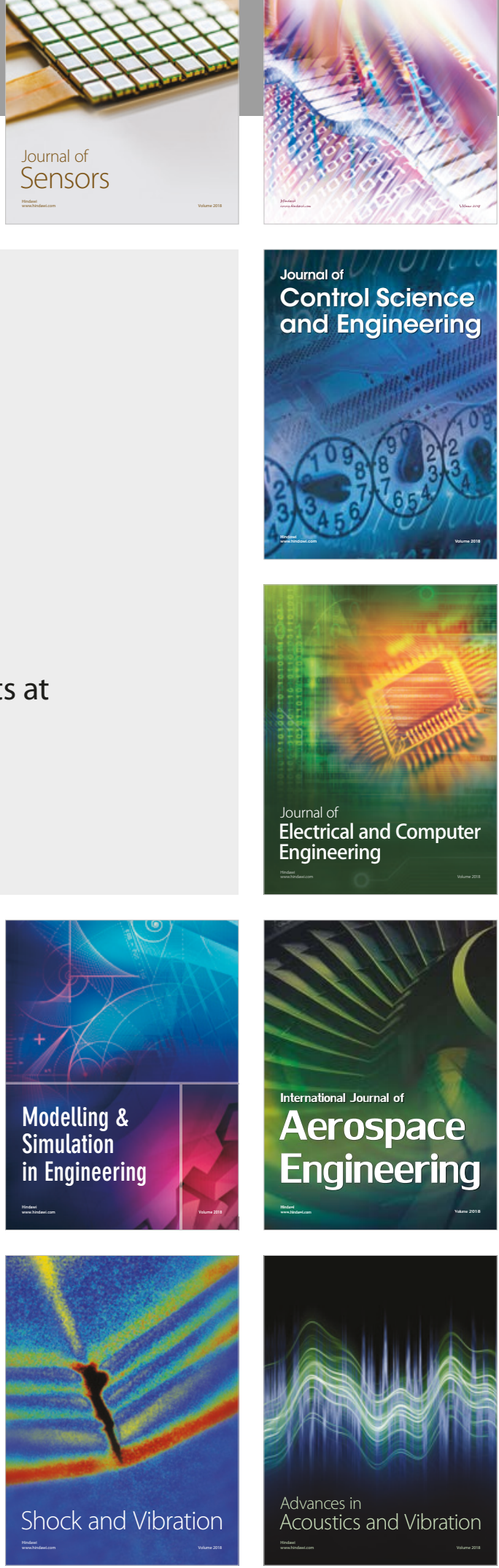